\title{
Defect-Aware Synthesis of Droplet-Based Microfluidic Biochips*
}

\author{
Tao $\mathrm{Xu}^{\dagger}$, Krishnendu Chakrabarty ${ }^{\dagger}$ and Fei $\mathrm{Su}^{\star}$ \\ ${ }^{\dagger}$ Department of Electrical and Computer Engineering \\ Duke University, Durham, NC \\ ${ }^{\ddagger}$ Intel Corporation, Folsom, CA
}

\begin{abstract}
Recent advances in microfluidics technology have led to the emergence of miniaturized biochip devices for biochemical analysis. A promising category of microfluidic biochips relies on the principle of electrowetting-on-dielectric, whereby discrete droplets of nanoliter volumes can be manipulated using an array of electrodes. As chemists adapt more bioassays for concurrent execution on such "digital" droplet-based microfluidic platforms, system integration, design complexity, and the need for defect tolerance are expected to increase rapidly. Automated design tools for defect-tolerant and multifunctional biochips are important for the emerging marketplace, especially for low-cost, portable, and disposable devices for clinical diagnostics. We propose a unified synthesis method that combines defect-tolerant architectural synthesis with defect-aware physical design. The proposed approach allows architectural-level design choices and defecttolerant physical design decisions to be made simultaneously. We use a large-scale protein assay and the polymerase chain reaction procedure as case studies to evaluate the proposed synthesis method. We also carry out simulations based on defect injection to evaluate the robustness of the synthesized biochip designs.
\end{abstract}

\section{Introduction}

Microfluidics technology and bioMEMS have seen tremendous growth in the past few years $[1,2,3]$. A major application driver for microfluidics is biochemical analysis based on fluidic processing in miniaturized devices, which are referred to in the literature as biochips. Biochips can be used for immunoassays, high-throughput sequencing, proteomic analysis involving proteins and peptides, and environmental toxicity monitoring. Another emerging application area for microfluidics-based biochips is clinical diagnostics, especially immediate point-of-care diagnosis of diseases [4].

A popular class of microfluidic biochips is based on continuous fluid flow in permanently-etched microchannels. Fluid flow in these devices is controlled either using micropumps and microvalves, or by electrical methods based on electrokinetics and electroosmosis [2]. An alternative category of microfluidic biochips relies on the principle of electrowetting-on-dielectric. Discrete droplets of nanoliter volume can be manipulated using an array of electrodes. Following the analogy of digital electronics, this technology is referred to as "digital microfluidics" [1]. Because each droplet can be controlled independently, these systems also have dynamic reconfigurability, whereby groups of unit cells in a microfluidic array can be reconfigured to change their functionality during the concurrent execution of a set of bioassays.

As chemists adapt more bioassays for concurrent execution on a digital microfluidic platform, system integration and design

\footnotetext{
*This work was supported in part by the National Science Foundation
} under grants IIS-0312352 and CCF-0541055. complexity are expected to increase steadily $[5,6]$. As in the case of integrated circuits (ICs), increase in density and area of microfluidics-based biochips will reduce yield, especially for new technology nodes. Low yield will deter large-scale and highvolume production, and it will increase production cost. It will take time to ramp up yield learning based on an understanding of defect types in such mixed-technology devices. Therefore, defect-tolerant designs are important for the emerging marketplace, especially for low-cost, portable, and disposable devices for clinical diagnostics.

Another reason for the importance of defect tolerance lies in the projected use of microfluidic biochips for safety-critical applications, e.g., patient health monitoring, neo-natal care, and the monitoring of environmental toxins. Therefore, defect tolerance must be integrated into the automated design tools to ensure high levels of system dependability. Despite the recent emergence of automated synthesis methods for biochips [6,7], defect tolerance has largely been overlooked in the literature.

In this paper, we propose a unified synthesis method that combines defect-tolerant architectural synthesis with defect-aware physical design. The proposed approach uses defect tolerance as a design criterion and it allows architectural-level design choices and defect-tolerant physical design decisions to be made simultaneously.

The rest of the paper is organized as follows. Section 2 provides an overview of digital microfluidic biochips. In Section 3, we discuss related prior work on biochip design automation. Section 4 presents an overview of the defect-oblivious unified synthesis method from [11]. In Section 5, we introduce a new criterion of evaluating defect tolerance for a digital microfluidic biochip and incorporate it in an enhanced version of the synthesis technique. In Section 6, we use a large-scale protein assay and a polymerase chain reaction (PCR) procedure as case studies to evaluate the proposed synthesis method. We also carry out simulations based on defect injection to evaluate the robustness of the synthesized biochip designs. Finally, conclusions are drawn in Section 7.

\section{Digital Microfluidic Biochips}

In this paper, we consider digital microfluidic biochips that utilize electrowetting to manipulate droplets of nanoliter volumes on a two-dimensional electrode array [1]. A unit cell in the array includes a pair of electrodes that acts as two parallel plates. The bottom plate contains a patterned array of individually controlled electrodes, and the top plate is coated with a continuous ground electrode. A droplet rests on a hydrophobic surface over an electrode, as shown in Figure 1. It is moved by applying a control voltage to an electrode adjacent to the droplet and, at the same time, deactivating the electrode just under the droplet. This electronic method of wettability control creates interfacial tension gradients that move the droplets to the charged electrode. Using the electrowetting phenomenon, droplets can be moved to any location on a two-dimensional array. 

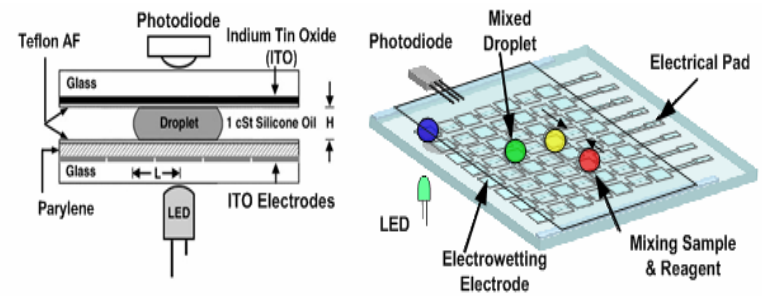

Figure 1: A digital microfluidic biochip.

By varying the patterns of control voltage activation, many fluidhandling operations such as droplet merging, splitting, mixing, and dispensing can be executed in a similar manner. For example, mixing can be performed by routing two droplets to the same location and then turning them about some pivot points [8]. The digital microfluidic platform offers the additional advantage of flexibility, referred to as reconfigurability, since fluidic operations can be performed anywhere on the array. Droplet routes and operation scheduling result are programmed into a microcontroller that drives electrodes in the array. In addition to electrodes, optical detectors such as LEDs and photodiodes are also integrated in microfluidic arrays to monitor colorimetric bioassays [3].

\section{Related Prior Work}

As in the case of IC design, biochip synthesis can be conceptually viewed as consisting of two stages, namely architectural synthesis and physical design. Architectural synthesis maps the behavioral model for a bioassay to a macroscopic structure of the biochip, which specifies scheduling and resource binding. Physical design generates the biochip layout, including the placement of microfluidic modules such as mixers, storage units, and integrated optical detectors.

Recent years have seen growing interest in the automated design and synthesis of microfluidic biochips $[5,6,7,9]$. One of the first published methods for biochip synthesis decouples high-level synthesis from physical design [10]. It is based on rough estimates for placement costs such as the areas of the microfluidic modules. These estimates provide lower bounds on the exact biochip area, since the overheads due to spare cells and cells used for droplet transportation are not known a priori. However, it cannot be accurately predicted if the biochip design meets system specifications, e.g., maximum allowable array area and upper limits on assay completion times, until both high-level synthesis and physical design are carried out. When design specifications are not met, time-consuming iterations between high-level synthesis and physical design are required. A link between these steps is especially necessary if defect tolerance is to be considered during synthesis.

More recently, a unified system-level synthesis method has been proposed for microfluidic biochips [11]. Based on parallel recombinative simulated annealing (PRSA) [12], this method allows users to describe bioassays at a high level of abstraction, and it automatically maps behavioral descriptions to the underlying microfluidic array. While [11] considers defect tolerance in the synthesis flow, its definition of defect tolerance is limited in scope. It is assumed in [11] that test and diagnosis procedures are used to locate defective cells in the microfluidic array after manufacture, following which the complete synthesis process has to be repeated to bypass defective cells. This approach is inefficient because defective cells tend to affect only a small number of microfluidic operations constituting a bioassay. Moreover, the synthesis method in [11] does not provide any quantitative metric to evaluate the defect tolerance of a synthesized biochip. Thus it does not allow

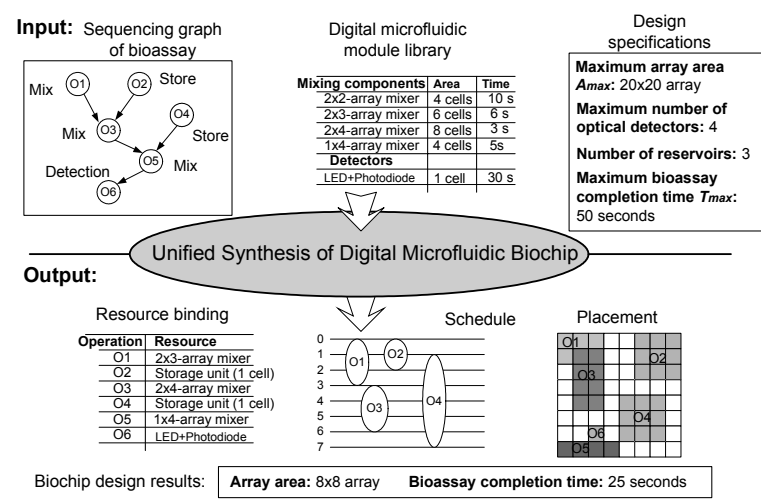

Figure 2. An example illustrating system-level synthesis [11].

the designer to consider defect tolerance as a selection criterion in the design process. In other words, defect tolerance in [11] is considered only after the device is manufactured, and it is not directly incorporated in the synthesis flow. For safety-critical applications involving healthcare and environmental monitoring, defect tolerance needs to be addressed early in the design flow.

\section{Unified Synthesis Methodology}

In this section, we provide an overview of the unified synthesis method presented in [11]. The design flow is illustrated in Figure 2. First, the different bioassay operations (e.g. mixing and dilution), and their mutual dependences are represented using a sequencing graph. Next, a combination of simulated annealing and genetic algorithms are used for unified resource binding, operation scheduling, and module placement. A chromosome is used to represent each candidate solution, i.e., a design point. In each chromosome, operations are randomly bound to resources. Based on the binding results, list scheduling is used to determine the start times of operations, i.e., each operation starts with a random latency after its scheduled time. Finally, a module placement is derived based on the resource binding and scheduling information.

A weighted sum of area- and time-cost is used to evaluate the quality of the design. The design is improved through a series of genetic evolutions based on PRSA. Test and diagnosis techniques to locate faulty cells in the microfluidic array have also been implemented using multi-step and adaptive Euler circuit-based testing procedures [13]. Post-manufacture defect tolerance can be achieved by labeling defective cells in the microfluidic array as "unavailable". The synthesis procedure is then restarted and the best solution for the biochips with unavailable cells is determined.

\section{Defect-Tolerant Synthesis}

Defect tolerance methods can be viewed as being either anticipatory, i.e., anticipate defect occurrences and design the system to be defect-resilient, or based on post-manufacture reconfiguration and re-synthesis. The focus of this paper is on defect-tolerant design, whereby we attempt we provide guarantees on correct bioassay operation even if the manufacturing process introduces defects. Instead of handling defects after they are detected, we attempt to achieve defect-tolerant mapping of bioassay protocols to the microfluidic array under broad assumptions of defect occurrences.

The synthesis method described in Section 4 suffers from two main drawbacks. First, it does not anticipate defect occurrences and consider defect tolerance in the synthesis flow. Instead, it relies on the availability of unused cells in the microfluidic array to avoid 


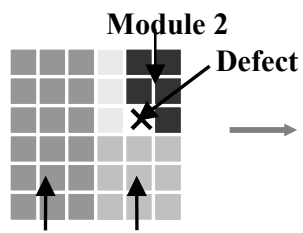

Module 1 Module 3

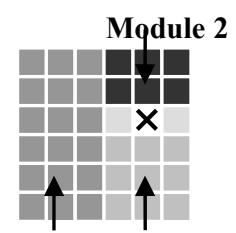

Module 1 Module 3
Figure 3. Example of partial reconfiguration.

defective cells that are located after manufacture. However, such a re-synthesis procedure might not be feasible because of lack of availability of spare cells. Moreover, the impact on assay completion time might be significant, and the upper limit on assay completion time might be violated. In such scenarios, the fabricated biochip must be discarded. A second drawback of defect-oblivious synthesis is that after defects are identified, the complete synthesis process must be repeated. Thus this approach imposes additional computation burden on the design and implementation process.

In this section, we propose a new method to incorporate defect tolerance in the unified synthesis flow for microfluidic biochips. A novel partial reconfiguration method is also presented to enhance defect tolerance after the device is manufactured.

\subsection{Defect Tolerance Index}

Defect tolerance of a synthesized biochip can be evaluated in terms of survivability, i.e., the capability to perform bioassays on a microfluidic array with defects. The Defect Tolerance Index (DTI), is defined as the probability that defect tolerance can be achieved via successful partial reconfiguration when the array contains defective cells [13]. Partial reconfiguration refers to the relocation only of the modules that contain defective cells; other modules are not affected. The relocated modules therefore "survive" through the defects (see Figure 3).

Assume that each cell in the microfluidic array has a independent failure probability $p$. The DTI $D(G)$ value for a layout $G$ can be estimated by multiplying the survival probabilities of all the modules, as follows [14]:

$$
D(G) \approx \Pi P s\left(M_{i}\right)=\Pi\left(1-f_{1}\left(M_{i}\right)+f_{1}\left(M_{i}\right) \times f_{2}\left(M_{i}\right)\right)
$$

where $M_{i}, i=1 \ldots N$, is a microfluidic module (e.g., mixer) contained in a given layout $G$, and $P s\left(M_{i}\right)$ is the survival probability of module $M$. Note that $f_{1}\left(M_{i}\right)$ is the probability that the module $M_{i}$ is faulty. It is determined by the equation $f_{1}\left(M_{i}\right)=1-p$ - $A\left(M_{i}\right)$, where $A\left(M_{i}\right)$ is the total number of cells contained in $M_{i}$. Finally, $f_{2}\left(M_{i}\right)$ is the probability that $M_{i}$ can be successfully reconfigured if it becomes faulty [13].

Now we incorporate DTI into the PRSA-based unified synthesis method. We first define layout vulnerability by $V=1-D$. Layouts with low vulnerability are likely to provide high probability of successful partial reconfiguration. To find such designs, we combine vulnerability with time- and area-cost to derive a new fitness function to control the PRSA-based procedure. The pseudocode for the defect-aware unified synthesis method is shown in Figure 4. Candidate designs with low survivability are discarded during evolution. Thus, the synthesis procedure anticipates defect occurrences and selects designs that allow reconfiguration of large number of modules, while meeting constraints on array size and bioassay processing time.

\subsection{Partial Reconfiguration and Partial Resynthesis}

Next we discuss how defects can be bypassed after manufacture.

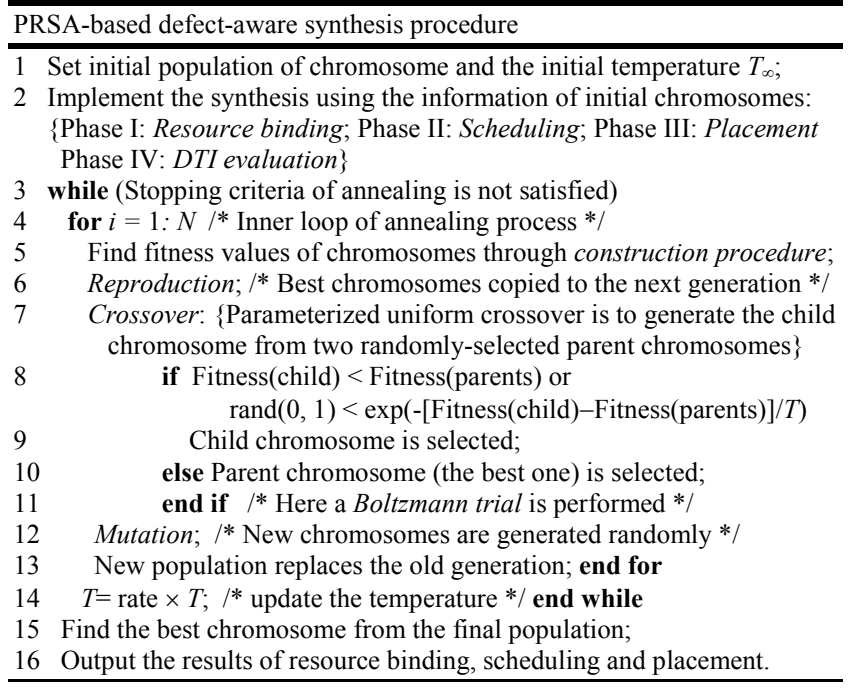

Figure 4: Pseudocode for the PRSA-based defect-aware synthesis

$$
\text { procedure. }
$$

In the defect-oblivious approach of [11], defect tolerance is achieved by complete resynthesis, which can be very time consuming. Here we propose an efficient method to achieve defect tolerance without the need for resynthesis. This method is based on the concept of partial reconfiguration, which was introduced in Section 5.1. If the number of defective cells is not excessive, most microfluidic modules on the array are not affected and they do not need not to be reconfigured. As discussed in the Section 5.1, the incorporation of defect tolerance in the design flow ensures a high probability of partial reconfigurability of the modules, i.e., it is very likely that the defective biochip can be made usable via partial reconfiguration.

For each affected module, we search the array for available defect free areas for partial reconfiguration. This can be accomplished fast, because the search space is restricted to the layouts in the modules' time duration. Once a module is relocated, the algorithm updates the corresponding layout and starts the search for the next module. Resources binding and scheduling results are not changed. Only the placement of defective modules is modified. Therefore, this method is much faster compared to a complete resynthesis procedure.

In some cases, there may be not enough defect free cells to carryout partial reconfiguration for some defective modules. We therefore introduce a new method called partial resynthesis. The key idea here is to truncate the bioassay and carry out resynthesis only for the modules that start later than the earliest-in-use defective module. Although this partial resynthesis procedure may take as much time as complete resynthesis in the worst case, i.e., if the first in-use module is defective and cannot be relocated, it is faster on average than the complete-resynthesis procedure.

Using these two methods, the complexity of doing postmanufacture processing for defect tolerance can be greatly reduced compared to resynthesis. The time-cost for a set of bioassays is also significantly decreased.

\section{Experimental Evaluation}

We evaluate the defect-tolerant synthesis method by using it to design a biochip for a real-life protein assay. Recently, the feasibility of performing a colorimetric protein assay on a digital microfluidic biochip has been successfully demonstrated [15] Based on the Bradford reaction [15], the protocol for a generic 


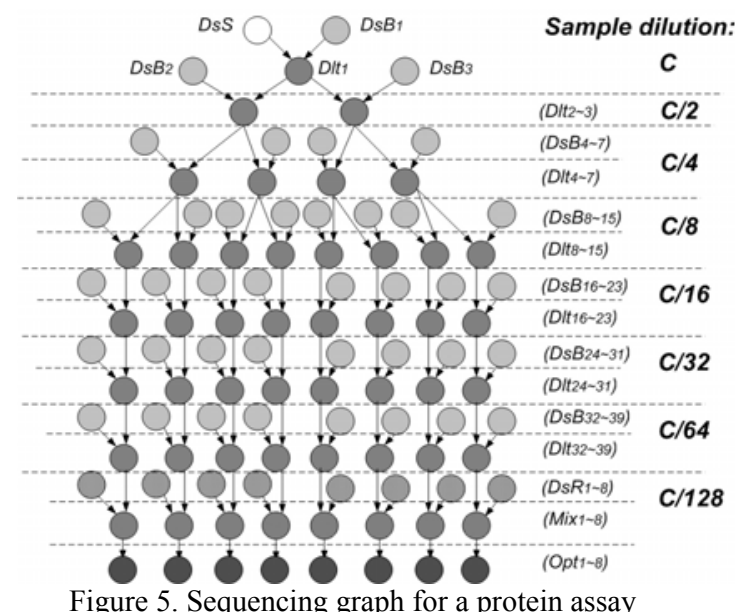

Figure 5. Sequencing graph for a protein assay

Table 1. Experimentally-characterized module library for synthesis.

\begin{tabular}{|c|l|c|}
\hline Operation & \multicolumn{1}{|c|}{ Resource } & Time (s) \\
\hline DsS;DsB; DsR & On-chip reservoir/dispensing port & 7 \\
\hline \multirow{5}{*}{ Dlt } & 2x2-array dilutor & 12 \\
\cline { 2 - 3 } & 2x3-array dilutor & 8 \\
\cline { 2 - 3 } & 2x4-array dilutor & 5 \\
\cline { 2 - 3 } & 4-electrode linear array dilutor & 7 \\
\hline \multirow{4}{*}{ Mix } & 2x2-array mixer & 10 \\
\cline { 2 - 3 } & 2x3-array mixer & 6 \\
\cline { 2 - 3 } & 2x4-array mixer & 3 \\
\cline { 2 - 3 } & 4-electrode linear array mixer & 30 \\
\hline Opt & LED+Photodiode & N/A \\
\hline Storage & Single cell & \\
\hline
\end{tabular}

droplet-based colorimetric protein assay is as follows. First, a droplet of the sample, such as serum or some other physiological fluid containing protein, is generated and dispensed into the biochip. Buffer droplets, such as $1 \mathrm{M} \mathrm{NaOH}$ solution, are then introduced to dilute the sample to obtain a desired dilution factor $(D F)$. This on-chip dilution is performed using multiple hierarchies of binary mixing/splitting phases, referred to as the interpolating serial dilution method [1]. The mixing of a sample droplet of protein concentration $C$ and a unit buffer droplet results in a droplet with twice the unit volume, and concentration $C / 2$. Splitting this large droplet results in two unit-volume droplets of concentration $C / 2$ each. Continuing this step in a recursive manner using diluted droplets as samples, an exponential dilution factor of $D F=2^{N}$ can be obtained in $N$ steps. After dilution, droplets of reagents, such as Coomassie brilliant blue G-250 dye, are dispensed into the chip, and they mix with the diluted sample droplets. Next the mixed droplet is transported to a transparent electrode, where an optical detector (e.g., a LED-photodiode setup) is integrated. The protein concentration can be measured from the absorbance of the products of this colorimetric reaction using a rate kinetic method [15].

Finally, after the assay is completed, all droplets are transported from the array to the waste reservoir. A sequencing graph model can be developed from the above protocol for a protein assay ( $D F$ $=128$ ), as shown in Figure 5. There are a total of 103 nodes in oneto-one correspondence with the set of operations in a protein assay, where $\mathrm{DsS}_{,} \mathrm{DsB}_{i}(i=1, \ldots, 39)$, and $\operatorname{DsR}_{i}(i=1, \ldots, 8)$ represents the generation and dispensing of sample, buffer and reagent droplets, respectively.

In addition, $\mathrm{Dlt}_{i}(i=1, \ldots, 39)$ denotes the binary dilution (including mixing/splitting) operations, $\operatorname{Mix}_{i}(i=1, \ldots, 8)$ represents the mixing of diluted sample droplets, and reagent droplets; $\operatorname{Opt}_{i}(i=1, \ldots, 8)$ denotes the optical detection of the

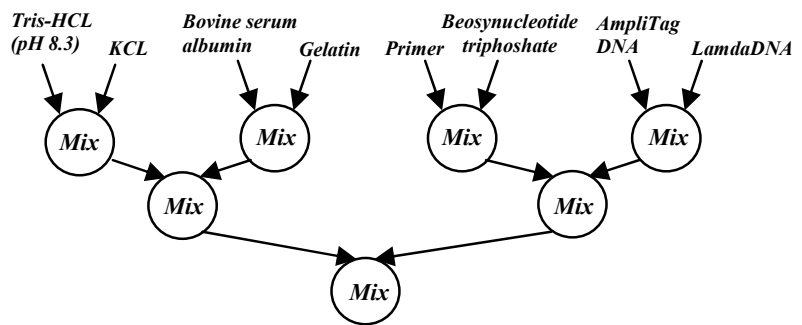

Figure 6. Sequencing graph for the mixing stage of PCR.

Table 2. Mixer library for PCR mixing stage.

\begin{tabular}{|l|l|c|}
\hline \multicolumn{1}{|c|}{ Hardware } & Module & Mixing time \\
\hline $2 \times 2$ electrode array & $4 \times 4$ cells & $10 \mathrm{~s}$ \\
\hline $2 \times 3$ electrode array & $4 \times 5$ cells & $6 \mathrm{~s}$ \\
\hline $2 \times 4$ electrode array & $4 \times 6$ cells & $3 \mathrm{~s}$ \\
\hline 4-electrode linear array & $3 \times 6$ cells & $5 \mathrm{~s}$ \\
\hline
\end{tabular}

droplets. Until the fourth step of a serial dilution, all diluted sample droplets are retained in the microfluidic array. After that stage, for each binary dilution step, only one diluted sample droplet is retained after splitting, while the other droplet is moved to the waste reservoir.

The basic operations for protein assay have been implemented on a digital microfluidic biochip $[1,15]$. Experiments indicate that the dispensing operation takes 7 seconds [1]. The operation times of various mixers have been found to be different [1]. A binary dilution operation can also be easily implemented by mixing of sample droplet followed by droplet splitting. Absorbance of the assay product can be measured using an integrated LEDphotodiode setup. Experiments indicate this absorbance measurement takes 30 seconds [15]. The microfluidic module library for a protein assay is shown in Table 1.

We also need to specify some design parameters for the biochip to be synthesized. As an example, we set the maximum microfluidic array size to be $10 \times 10$ cells, and the maximum allowable completion time for the protein assay to be 400 seconds. We assume that there is only one on-chip reservoirs/dispensing port available for sample fluids, but there are two such ports for buffer fluids, two for reagent fluids, and one for waste fluids. Finally, we assume that at most four optical detectors can be integrated into this biochip.

A second bioassay, namely the mixing stages of the polymerase chain reaction (PCR) is also used in this paper. PCR is one of the most commonly used procedure for DNA analysis. It is used for rapid enzymatic amplification of specific DNA exponentially using temperature cycles. Recently, the feasibility of performing dropletbased PCR on digital microfluidics-based biochips has been successfully demonstrated [16]. Here we use the mixing stage of PCR as an example to evaluate the defect tolerance capability of defect-oblivious and defect-aware synthesis methods. Its assay protocol can be modeled by a sequencing graph, as shown in Figure 6. The resource library is shown in Table 2. The data for the operation times associated with the different modules are obtained from real-life experiments. Unlike the protein assay, no design constraints (area-cost, time-cost) are provided for the PCR assay.

We first use the defect-oblivious PRSA-based algorithm of Section 4 to find a desirable solution for the protein assay that satisfies design specifications. The solution thus obtained yields a biochip design with a $9 \times 9$ microfluidic array and an assay completion time of 363 seconds. Next we investigate defecttolerant synthesis method using the procedure of Figure 4 . The 


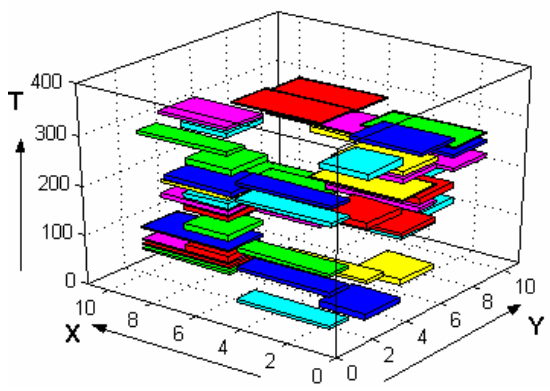

Figure 7. A 3-D model illustrating defect tolerant synthesis results for the protein assay.

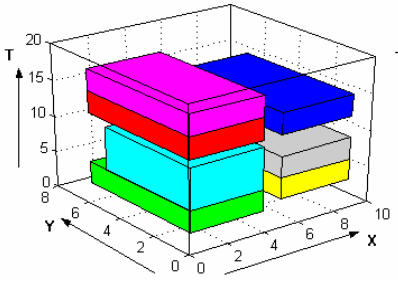

(a)

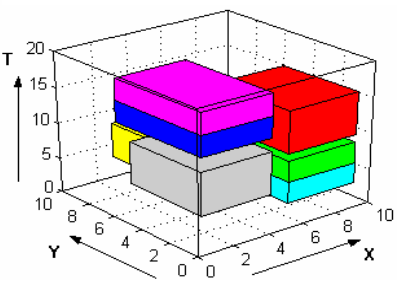

(b)
Figure 8. 3-D model illustrating the synthesis results of a digital microfluidic biochip for PCR mixing stage using (a) [11] (b) the proposed method.

solution obtained yields a biochip design with a $10 \times 10$ microfluidic array and completion time of 377 seconds. We illustrate the synthesis results, i.e., assay operation schedule and module placement, using a 3-D box model shown in Figure 7. Each microfluidic module is represented as a 3-D box, the base of which denotes the rectangular area of the module and the height denotes the time-span of the corresponding assay operation. The projection of a 3-D box on the X-Y plane represents the placement of this module on the microfluidic array, while the projection on the $\mathrm{T}$ axis (time axis) represents the schedule of the assay operation. Note that all these boxes are contained in a bin of size $X_{\max } \times Y_{\max } \times T_{\max }$, where $A_{\max }=X_{\max } \times Y_{\max }$; this implies that this design satisfies the specifications of array area and assay completion time. Moreover, there is no overlap between these boxes, thereby avoiding a violation of resource constraints. In addition, the synthesis results also determine the locations of integrated optical detectors. Transparent electrodes for optical detection are used in the microfluidic array. We can further integrate optical detectors as well as on-chip reservoirs/dispensing ports into the microfluidic array to form a complete digital microfluidic biochip for the protein assay.

Although the solution from the enhanced algorithm leads to slightly higher area and assay time than obtained in [11], this design leads to a DTI value of 0.9495 , which implies that almost $95 \%$ of the modules can be reconfigured if they are affected by defects. This is a considerable improvement over the DTI value of 0.0006 obtained using the method presented in [11]. Note that in the design obtained in [11], since DTI $\approx 0$, almost none of the modules can be relocated using partial reconfiguration. The improvement also implies a significant reduction in time-cost for achieving defect tolerance. While complete resynthesis for the protein assay takes 40 minutes of CPU time on a $1.86 \mathrm{GHz}$ Pentium-M PC with $1 \mathrm{G}$ of RAM, partial reconfiguration takes less than 10 seconds.

The results also show that defect-tolerant synthesis does not necessarily lead to significant time-cost increase. For example, the time-cost just goes only from 363 to 377 seconds, an increase of less than $3 \%$.

Next, we apply the defect-oblivious method in [11] and our proposed defect-aware synthesis tools to the PCR assay. Results are derived for both synthesis methods are shown in the 3-D box in Figure 8. The defect-oblivious method of [11] leads to a biochip design with a $9 \times 7$ microfluidic array and an operation time of 16 seconds while the defect-aware method yields a design with a $9 \times 9$ array and an operation time of 18 seconds.

From the results for both the two assays, i.e., protein assay and PCR mixing stage, we conclude that the defect-aware method usually leads to a slightly larger array area and time-cost compared to the defect-oblivious method. This is reasonable because defectaware method has to consider reconfiguration a priori. Therefore, in ideal situations, i.e., for defect free cases, the method in [11] is a better choice because of the compactness and time efficiency of the design. However, in practice, when defects are likely, the defectaware methods offers key advantages over [11] in many aspects, as highlighted next in Section 6.2.

\subsection{Results for Defect Injection \\ 6.2.1 Protein Assay Example}

We next evaluate the defect tolerance of the synthesized design by injecting random defects. A design is deemed to be robust if the injected defects can be bypassed by partial reconfiguration. Defects can be classified based on their impact on bioassay functionality.

The first category includes defects that affect only the unused cells in the array. As the biochip functionality is not compromised, these defects are referred to as benign. The second category refers to defects that cause significant "fragmentation" of the array, whereby it is no longer possible to relocate a microfluidic module to another part of the array due to lack of availability of defect-free cells. These defects are referred to as catastrophic. The third category includes defects that are neither benign nor catastrophic. The microfluidic array can be reconfigured for such defects, hence we refer to these defects as repairable.

A biochip that contains only benign defects is placed in Group I. A biochip that contains catastrophic defects is placed in Group II. Finally, a biochip that contains only repairable and benign defects is placed in Group III. Let $N_{t}$ be the total number of biochips in a representative sample, and let $N_{i}$ be the number of biochips in group I, $1<i<3$. Clearly $N_{1}+N_{2}+N_{3}=N_{t}$. We next define two ratios related to the defect tolerance capability of the synthesized biochip: (i) robustness index $r=\left(N_{1}+N_{3}\right) / N_{t}$; (ii) failure index $f=N_{2} / N_{t}$. The goal of defect-aware synthesis is to maximize $r$ and minimize $f$.

Resynthesis must be carried out for biochips in Group II, i.e., for biochips that suffer from catastrophic defects. Let the bioassay completion time before (after) resynthesis be $T_{1}\left(T_{2}\right)$. We define the time degradation $t d$ as follow: $t d=\left(T_{2}-T_{1}\right) / T_{1}$. Another goal of defect-aware synthesis is to minimize $t d$.

We next take 100 simulated samples of a microfluidic biochip synthesized for the protein assay using the proposed method and using [11]. In each case, we randomly inject defects by assuming that each unit cell is defective with probability $p(p=0.01,0.05$, 0.1 in our experiments). We then determine the ratios $r, f$, and $t d$ for both methods. The results are shown in Table 3.

As mentioned in Section 6.1, defect-aware synthesis slightly increases assay time and array area for the protein assay. The key advantage is that it leads to a high DTI value of 0.9495 , which implies that almost all modules, once defects are defected, can be reconfigured. This is a significant improvement compared to the DTI value of 0.0006 for [11], where for most modules, defect occurrence is catastrophic and resynthesis has to be carried out. 
Table 3. Defect tolerance for [11] and the proposed method for the protein assay.

\begin{tabular}{|l|l|l|l|l|l|l|}
\hline & $\begin{array}{l}\text { DTI } \\
\text { value }\end{array}$ & $\begin{array}{l}\text { Assay } \\
\text { Time (s) }\end{array}$ & $\begin{array}{l}\text { Array } \\
\text { Area }\end{array}$ & $r$ & $f$ & $t d$ \\
\hline Method in [11] & 0.0006 & 363 & $9 \times 9$ & 0.07 & 0.93 & 1.45 \\
\hline Proposed method & 0.9495 & 377 & $10 \times 10$ & 0.88 & 0.12 & 1.14 \\
\hline & $\begin{array}{l}\text { DTI } \\
\text { value }\end{array}$ & $\begin{array}{l}\text { Assay } \\
\text { Time (s) }\end{array}$ & $\begin{array}{l}\text { Array } \\
\text { Area }\end{array}$ & $r$ & $f$ & $T d$ \\
\hline Method in [11] & 0.0006 & 363 & $9 \times 9$ & 0.15 & 0.85 & 1.34 \\
\hline Proposed method & 0.9495 & 377 & $10 \times 10$ & 0.91 & 0.09 & 1.08 \\
\hline \multicolumn{7}{|c|}{ (b) $p=0.05$} \\
\hline
\end{tabular}

$$
\text { (c) } p=0.01
$$

Table 4. Defect tolerance for [11] and the proposed method for the PCR assay.

\begin{tabular}{|l|l|l|l|l|l|l|}
\hline & $\begin{array}{l}\text { DTI } \\
\text { Value }\end{array}$ & $\begin{array}{l}\text { Assay } \\
\text { Time (s) }\end{array}$ & $\begin{array}{l}\text { Array } \\
\text { Area }\end{array}$ & $r$ & $f$ & $t d$ \\
\hline Method in [11] & 0.0136 & 16 & $9 \times 7$ & 0.11 & 0.89 & 2.64 \\
\hline Proposed method & 0.8921 & 18 & $9 \times 9$ & 0.90 & 0.20 & 1.93 \\
\hline & $\begin{array}{l}\text { DTI } \\
\text { Value }\end{array}$ & $\begin{array}{l}\text { Assay } \\
\text { Time (s) }\end{array}$ & $\begin{array}{l}\text { Array } \\
\text { Area }\end{array}$ & $r$ & $f$ & $t d$ \\
\hline Method in [11] & 0.0136 & 16 & $9 \times 7$ & 0.19 & 0.81 & 1.92 \\
\hline Proposed method & 0.8921 & 18 & $9 \times 9$ & 0.93 & 0.11 & 1.31 \\
\hline \multicolumn{7}{|c|}{ (b) $p=0.05$} \\
\hline & $\begin{array}{l}\text { DTI } \\
\text { value }\end{array}$ & $\begin{array}{l}\text { Assay } \\
\text { Time (s) }\end{array}$ & $\begin{array}{l}\text { Array } \\
\text { Area }\end{array}$ & $r$ & $f$ & $t d$ \\
\hline Method in [11] & 0.0136 & 16 & $9 \times 7$ & 0.28 & 0.72 & 1.47 \\
\hline Proposed method & 0.8921 & 18 & $9 \times 9$ & 0.98 & 0.02 & 1.00 \\
\hline
\end{tabular}

$$
\text { (c) } p=0.01
$$

This improvement is verified by the comparison of failure ratio $(f)$, robustness index $(r)$ and time degradation $(t d)$ from Table 3.

For all the three value of $p$, defect-aware synthesis results in a higher value of $r$ and a considerably lower $f$. Moreover, the defectaware biochip design also provides a much lower value of $t d$, which implies that for resynthesized biochips, the performance is compromised much less. Since the original time-cost for the two methods are comparable, the difference in $t d$ is therefore even more significant. Moreover, $t d$ falls more sharply for smaller values of $p$ for the defect-aware synthesis design. Therefore, for low defect probabilities, as is often the cases for mature manufacturing processes, the proposed defect tolerant synthesis method allows resynthesis in the case of catastrophic defects with lower time-cost increase. This feature is often required by many biochip applications.

\subsubsection{PCR Example}

Next, defect injection is carried out for the two biochip designs determined using defect-oblivious and defect-aware methods for the PCR example. Due to the relative lower complexity of the PCR assay, the number of simulation runs is increased to 1000 for smoother averaging of experimental parameters. The results are listed in Table 4.

As in the case of protein assay, a significant enhancement in defect tolerance is observed. In particular, for biochips with high failure probability, e.g., $p=0.1$, defect-aware synthesis design reduces the failure index from 0.89 to 0.20 , an improvement of
$78 \%$. Defect-aware synthesis also leads to much lower time degradation. This advantage is even more prominent for biochips with small failure probability. As shown in Table 4(c), defect tolerant synthesis leads to negligible time degradation.

\section{Conclusion}

In this paper, we have presented a new defect aware synthesis methodology for droplet-based microfluidic biochips. The synthesis procedure, which is based on parallel recombinative simulated annealing, unifies defect tolerance with the scheduling of bioassay operations, resource binding, and module placement. By introducing a new criterion to evaluate defect tolerance, we have shown how the proposed synthesis method can lead to designs that can tolerant manufacturing defects. Partial resynthesis and partial reconfiguration have been proposed to address manufacturing defects. Real-life examples of a protein assay based on the Bradford reaction, and PCR have been used to evaluate the effectiveness of the synthesis procedure. Finally, we have reported simulation results based on defect injection for both the protein assay and the PCR example. Our results show that defect-aware synthesis leads to higher robustness, lower failure ratio, and lower performance degradation in the presence of defects. This work is expected to facilitate the automated design of biochips with guaranteed high levels of defect tolerance. In this way, the biochip user can concentrate on the development of nano- and micro-scale bioassays, leaving cumbersome implementation details to the synthesis tools.

\section{References}

[1] R. B. Fair et al., "Electrowetting-based on-chip sample processing for integrated microfluidics", Proc. IEDM, 2003, pp. 32.5.1-32.5.4.

[2] E. Verpoorte and N. F. De Rooij, "Microfluidics meets MEMS", Proc. IEEE, 2003, vol. 91, pp. 930-953.

[3] J. Zeng and T. Korsmeyer, "Principles of droplet electrohydrodynamics for lab-on-a-chip", Lab on a Chip, 2004, vol. 4, pp. 265-277.

[4] T. H. Schulte et al., "Microfluidic technologies in clinical diagnostics", Clinica Chimica Acta, 2002, vol. 321, pp. 1-10.

[5] F. Su, K. Chakrabarty and R. B. Fair, "Microfluidics-based biochips: technology issues, implementation platforms, and design automation challenges", IEEE Trans. CAD, February 2006, vol. 25, pp. 211-223.

[6] K. Chakrabarty and J. Zeng, "Design automation for microfluidicsbased biochips", ACM Journal on Emerging Technologies in Computing Systems, December 2005, vol. 1, pp. 186-223.

[7] P.-H. Yuh et al., "Placement of digital microfluidic biochips using the T-tree formulation", Proc. DAC, 2006, pp. 931- 934.

[8] P. Y. Paik et al., "Rapid droplet mixers for digital microfluidic systems", Lab on a Chip, 2003, vol. 3, pp. 253-259.

[9] T. Mukherjee, "Design automation issues for biofluidic microchips" Proc. ICCAD, 2005, pp. 463-470.

[10] F. Su and K. Chakrabarty, "Architectural-level synthesis of digital microfluidics-based biochips", Proc. ICCAD, 2004, pp. 223-228.

[11] F. Su and K. Chakrabarty, "Unified high-level synthesis and module placement for defect-tolerant microfluidic biochips", Proc. DAC, 2005, pp. 825-830.

[12] S. W. Mahfoud and D. E. Goldberg, "Parallel recombinative simulated annealing: a genetic algorithm," Parallel Computing, 1995, vol. 21, pp. 128.

[13] F. Su et al, "Defect-oriented testing and diagnosis of digital microfluidics-based biochips", Proc.ITC, 2005, pp. 487-496.

[14] F. Su and K. Chakrabarty, "Module placement for fault-tolerant microfluidics-based biochips", ACM Transactions on Design Automation of Electronic Systems, 2006, vol. 11, pp. 682-710.

[15] V. Srinivasan et al., "Protein stamping for MALDI mass spectrometry using an electrowetting-based microfluidic platform", Proc. SPIE, vol. 5591, 2004, pp. 26-32.

[16] C. G. J. Schabmueller et al , "Closed chamber PCR chips for DNA amplification”, Eng. Sci. Educ. J., 2000, vol. 9, no. 6, pp. 259-264. 